\title{
Recurrent Laryngeal Nerve Monitoring During Esophagectomy and Mediastinal Lymph Node Dissection: Reply
}

\author{
Dieter Cadosch • Hans Gelpke
}

Published online: 21 July 2012

(C) Société Internationale de Chirurgie 2012

To the Editor,

We are very grateful for the comments and the constructive suggestions made by Schmidt and co-workers regarding our recent article [1]. It is our opinion that the proposed method is a valuable alternative to our protocol.

Since 2008 we routinely use the published method for recurrent laryngeal nerve monitoring during esophagectomy, left sided lung resections with mediastinal lymph node dissection, and cases of right-sided lung resections with high right-sided or additional left-sided mediastinal lymph node dissection. We agree that maintaining the position of the electrode can be challenging with a doublelumen endotracheal tube when the patient needs to be repositioned after intubation. This has been the case in the past. In the meantime we adapted our protocol and replaced the initial electrode $(1.5 \mathrm{~cm}$ long) by a longer one $(4.5 \mathrm{~cm}$ long) (Dragonfly laryngeal electrode, Spes Medica S.r.l., Battipaglia, Italy). With the longer electrode we were able to identify the recurrent laryngeal nerve (RLN) and monitor a nerve signal in all patients independent of the pathology present or the type of surgery employed.
Instead of using an electrode on a double-lumen tube, Schmidt and co-workers propose using a single-lumen tube in combination with a bronchial blocker. This allows repositioning of the endotracheal tube if the EMG signal is absent, without interfering with single lung ventilation. However, deflating the unventilated lung may be more difficult with single lung ventilation in combination with a bronchus blocker [2].

\section{References}

1. Gelpke H, Grieder F, Decurtins M, Cadosch D (2010) Recurrent laryngeal nerve monitoring during esophagectomy and mediastinal lymph node dissection. World J Surg 34:2379-2382. doi: 10.1007/s00268-010-0692-0

2. Narayanaswamy M, McRae K, Slinger P et al (2009) Choosing a lung isolation device for thoracic surgery: a randomized trial of three bronchial blockers versus double-lumen tubes. Anesth Analg 108:1097-1101

D. Cadosch $(\square)$

Clinic of Trauma Surgery, University Hospital Zurich,

Raemistrasse 100, 8091 Zurich, Switzerland

e-mail: dieter.cadosch@usz.ch

H. Gelpke

Department of Surgery, Kantonsspital Winterthur,

Brauerstrasse 15, 8401 Winterthur, Switzerland 